\title{
A NEW CLASS OF DISCRETIZATION METHODS FOR THE SOLUTION OF LINEAR DIFFERENTIAL-ALGEBRAIC EQUATIONS WITH VARIABLE COEFFICIENTS
}

\author{
PETER KUNKEL * AND VOLKER MEHRMANN †
}

\begin{abstract}
We discuss new discretization methods for linear differential-algebraic equations with variable coefficients. We introduce numerical methods to compute the local invariants of such differential-algebraic equations that were introduced by the authors in a previous paper. Using these quantitities we are able to determine numerically global invariances like the strangeness index, which generalizes the differentiation index for differential-algebraic equations that in particular include undetermined solution components. Based on these methods we then obtain regularization schemes, which allow us to employ general solution methods. The new methods are tested on a number of numerical examples.
\end{abstract}

Key words. differential-algebraic equations, canonical forms, backward difference formulas, least square regularization, singular pencils, strangeness index

AMS(MOS) subject classifications. 65L05

1. Introduction. We discuss the numerical solution of linear differentialalgebraic equations (DAE's) with variable coefficients

$$
E(t) \dot{x}(t)=A(t) x(t)+f(t), t \in[\underline{t}, \bar{t}],
$$

where $E, A \in C\left([\underline{t}, \bar{t}], \mathbf{C}^{n, n}\right), f \in C\left([\underline{t}, \bar{t}], \mathbf{C}^{n}\right)$ together with an initial condition

$$
x\left(t_{0}\right)=x_{0}, t_{0} \in[\underline{t}, \bar{t}], x_{0} \in \mathbf{C}^{n} .
$$

Here $C^{\ell}\left([\underline{t}, \bar{t}], \mathbf{C}^{n}\right)$ denotes the set of $\ell$-times continuously differentiable functions from the interval $[\underline{t}, \bar{t}]$ to the $n$-dimensional complex vector space $\mathbf{C}^{n}$.

Definition 1. A function $x:[\underline{t}, \bar{t}] \rightarrow \mathbf{C}^{n}$ is called a solution of (1) if $x \in$ $C^{1}\left([\underline{t}, \bar{t}], \mathbf{C}^{n}\right)$ and $x$ satisfies (1) pointwise.

It is called a solution of the initial value problem (1), (2) if $x$ is a solution of (1) and $x$ satisfies (2).

An initial condition (2) is called consistent if the corresponding initial value problem is solvable, i.e. has at least one solution.

Note that the notion of solvability differs from others used in the literature (see e.g. $[2,3]$ or $[20]$ for an overview). The notion used here is a straightforward generalization of the one known from ordinary differential equations.

In a recent paper, Kunkel/Mehrmann [23] have introduced a new canonical form for (1) and corresponding invariants under equivalence transformation with variable transformation matrices. Using these results they have given existence and uniqueness results for the solution of (1). The most important invariant in their considerations is the so called strangeness index, which generalizes the differentiation index $([3,6,13])$ for systems with undetermined components which occur, for example, in the solution of linear quadratic optimal control problems and differential-algebraic Riccati equations, see e.g. [21, 22, 27]. It is known that most of the standard integration methods

\footnotetext{
* Fachbereich Mathematik, Carl von Ossietzky Universität, Postfach 2503, D-26111 Oldenburg, Fed. Rep. Germany

$\dagger$ Fakultät für Mathematik, TU Chemnitz-Zwickau, PSF 964, D-09009 Chemnitz, Fed. Rep. Germany.
} 
for general DAE's require the system to have differentiation index not higher than one, which corresponds to a vanishing strangeness index, see [23]. If this condition is not valid or if the DAE has undetermined components, the standard methods as implemented in codes like DASSL of Petzold [28] or LIMEX of Deufhard/Hairer/Zugck [10] often fail.

In this paper we introduce numerical methods to compute the strangeness index as well as other invariants and, based on this information, to extract a strangeness free DAE. In the case the DAE is found to be uniquely solvable for consistent initial values we then can compute a consistent initial value and apply the well-known integration schemes for DAE's, see $[2,17,19]$. In the case of undetermined solution components we use a least squares approach to fix a solution. Least squares approaches are also used in $[1,5,6,12]$. Their approaches, in contrast to the approach taken here, have in common that they treat uniquely solvable higher index problems by adding extra equations and solving the so obtained overdetermined system via least squares. In our approach the invariants as well as the strangeness free DAE are obtained from the original DAE and its higher derivatives. The higher derivatives are also used in an approach introduced by Campbell, see $[3,5,6,8]$ aiming in the determination of the so-called underlying ordinary differential equation which, if defined, is then uniquely solvable together with the initial value (2). Note, however, that this underlying ODE does no longer reflect the problem of consistent initial values nor may it exhibit the stability properties of the original DAE (see e.g. [25, 26]) and additional work is required to avoid the occurence of wrong dynamics, see [4]. Recently another new approach for the treatment of inconsistent initial values has been suggested in [9]. In the present paper, the extracted strangeness free DAE will bear the same solution behaviour as the original DAE. These results are closely related to the normal form introduced in [23] and can be obtained via sole application of unitary transformations. Because we make extent use of the results of [23], we briefly summarize the results obtained there. We begin with two equivalence relations that play a central role in the theory of (1). The first one describes all possible non-constant regular transformations of (1).

Definition 2. Two pairs of matrix functions $\left(E_{i}(t), A_{i}(t)\right)$ with $E_{i}, A_{i} \in$ $C\left([\underline{t}, \bar{t}], \mathbf{C}^{n, n}\right), i=1,2$ are called equivalent if there exist $P \in C\left([\underline{t}, \bar{t}], \mathbf{C}^{n, n}\right)$ and $Q \in C^{1}\left([\underline{t}, \bar{t}], \mathbf{C}^{n, n}\right)$ with $P(t), Q(t)$ nonsingular for all $t \in[\underline{t}, t]$ such that the matrix pairs transform as

$$
\left(E_{2}(t), A_{2}(t)\right)=P(t)\left(E_{1}(t), A_{1}(t)\right)\left[\begin{array}{cc}
Q(t) & -\dot{Q}(t) \\
0 & Q(t)
\end{array}\right] .
$$

Since we want to develop numerical methods, we also need a counterpart of this equivalence that can be obtained locally at a fixed point. At a fixed point $t \in[\underline{t}, \bar{t}]$ we can choose $Q(t)$ and $\dot{Q}(t)$ independently (see [14, 23]), so we obtain the following definition of equivalence for constant pencils:

Definition 3. Two pairs of matrices $\left(E_{i}, A_{i}\right), E_{i}, A_{i} \in \mathbf{C}^{n, n}, \quad i=1,2$ are called locally equivalent if there are matrices $P, Q, B \in \mathbf{C}^{n, n}$ with $P, Q$ nonsingular such that

$$
\left(E_{2}, A_{2}\right)=P\left(E_{1}, A_{1}\right)\left[\begin{array}{cc}
Q & -B \\
0 & Q
\end{array}\right] .
$$

Note that this is not a transformation that can be used in the DAE, since we cannot transform $x(t)$ and $\dot{x}(t)$ independently. 
For this local equivalence the following normal form is proved in [23]:

Theorem 1.1. Let $E, A \in \mathbf{C}^{n, n}$. Consider the following matrices and the spaces spanned by their columns:

(a) $T$ basis of kernel $E$

(b) $Z$ basis of corange $E=$ kernel $E^{*}$

(c) $T^{\prime}$ basis of cokernel $E=$ range $E^{*}$

(d) $V$ basis of corange $\left(Z^{*} A T\right)$.

Then the quantities (with the convention $\operatorname{rank} \emptyset=0$ )
(a) $r=\operatorname{rank} E$
(rank)
(b) $\quad a=\operatorname{rank}\left(Z^{*} A T\right)$
(algebraic part)
(c) $s=\operatorname{rank}\left(V^{*} Z^{*} A T^{\prime}\right)$
(strangeness)
(d) $\quad d=r-s$
(differential part)
(e) $u=n-r-a-s \quad$ (undetermined part)

are well-defined and invariant under (4) and $(E, A)$ is equivalent to the canonical form

$$
\left(\left[\begin{array}{ccccc}
I_{s} & 0 & 0 & 0 & 0 \\
0 & I_{d} & 0 & 0 & 0 \\
0 & 0 & 0 & 0 & 0 \\
0 & 0 & 0 & 0 & 0 \\
0 & 0 & 0 & 0 & 0
\end{array}\right],\left[\begin{array}{ccccc}
0 & 0 & 0 & 0 & 0 \\
0 & 0 & 0 & 0 & 0 \\
0 & 0 & I_{a} & 0 & 0 \\
I_{s} & 0 & 0 & 0 & 0 \\
0 & 0 & 0 & 0 & 0
\end{array}\right]\right) \begin{gathered}
s \\
d \\
a \\
s \\
u
\end{gathered} .
$$

Note that the characteristic values $r, a, s, d, u$ are computable via three rank decisions that can be obtained for example via singular value decompositions.

Applying now the results for the local canonical form (7) to equation (1) one obtains functions

$$
r, a, s:[\underline{t}, \bar{t}] \rightarrow\{0, \ldots, n\} .
$$

Without any further restrictions, (1) in particular includes singular ordinary differential equations and bifurcation problems which are hard problems by themselves. These types of problems are directly connected with discontinuities in the above functions $r, a, s$. For these reasons, we exclude such phenomena by assuming

$$
r(t) \equiv r, a(t) \equiv a, s(t) \equiv s
$$

throughout the rest of this paper. Note, however, that this assumption can be partially relaxed as indicated in some very recent papers [7, 29]. Points where theses quantities have jumps, nonetheless, may be of physical importance and thus these should be detected. In the numerical procedure that we present, we identify these points. A general classification is currently under investigation.

For the equivalence relation (3) using $\dot{Q}$ instead of an arbitrary $B$ one cannot eliminate as many elements of the pair as in (7). In [23] it is shown that for $E, A$ in (1) sufficiently smooth and satisfying (8), equation (1) is equivalent to the system of differential-algebraic equations

$$
\begin{array}{rlrl}
\text { (a) } & \dot{x}_{1}(t) & =A_{12}(t) x_{2}(t)+A_{14}(t) x_{4}(t)+A_{15}(t) x_{5}(t)+f_{1}(t) \\
\text { (b) } & \dot{x}_{2}(t) & =A_{24}(t) x_{4}(t)+A_{25}(t) x_{5}(t)+f_{2}(t) \\
(c) & 0 & =x_{3}(t)+f_{3}(t) \\
\text { (d) } & 0 & =x_{1}(t)+f_{4}(t) \\
\text { (d) } & 0 & & =f_{5}(t) .
\end{array}
$$


Differentiating equation (9d) and inserting it in (9a), we obtain the system

$$
\begin{array}{rlrl}
\text { (a) } & & 0 & =A_{12}(t) x_{2}(t)+A_{14}(t) x_{4}(t)+A_{15}(t) x_{5}(t)+f_{1}(t)+\dot{f}_{4}(t) \\
\text { (b) } & \dot{x}_{2}(t) & =A_{24}(t) x_{4}(t)+A_{25}(t) x_{5}(t)+f_{2}(t) \\
\text { (c) } & 0 & =x_{3}(t)+f_{3}(t) \\
\text { (d) } & 0 & =x_{1}(t)+f_{4}(t) \\
\text { (e) } & 0 & = & f_{5}(t) .
\end{array}
$$

Applying now repeatedly the transformation to the forms (9) and then (10) one obtains an inductive definition of a sequence of pairs of matrix functions $\left(E_{i}(t), A_{i}(t)\right), \quad i \in \mathbf{N}_{0}$, starting with $\left(E_{0}(t), A_{0}(t)\right)=(E(t), A(t))$ and $\left(E_{i+1}(t), A_{i+1}(t)\right)$ is derived from $\left(E_{i}(t), A_{i}(t)\right)$ by one step of this procedure.

Here we assume (8) for every occuring pair of matrices. Connected with this sequence, we then have sequences $r_{i}, a_{i}, s_{i}, d_{i}, u_{i}, i \in \mathbf{N}_{0}$ of nonnegative integers. These sequences are characteristic for the given DAE, that is, they do not depend on the specific way they are obtained. The assumptions of all characteristic values being constant on the whole interval of consideration may appear to be rather restrictive compared with other existence and uniqueness investigations. But observe that we can at once relax these assumptions by allowing an arbitrary matrix in front of $\dot{x}_{1}$ in (9a) without disturbing the following steps of the above procedure. However, a complete discussion of possible phenomena connected with rank changes including an examination what kind of rank changes do not affect the presented results are beyond the scope of this paper.

Since $\operatorname{rank} E_{i+1}(t)=\operatorname{rank} E_{i}(t)-s_{i}$, there must be an index $i$ such that $s_{i}=0$. Having once obtained $s_{i}=0$, the above process becomes stationary. Therefore the quantity

$$
m=\min \left\{i \in \mathbf{N}_{0} \mid s_{i}=0\right\}
$$

is well-defined. As $m$ is characteristic for a given DAE of the form (1) with the above properties and gives the number of differentiations we must apply to reach a system with vanishing strangeness, we call $m$ the strangeness index of the DAE. Note that in contrast the differentiation index $k$ is defined to be the number of differentiations we must apply to be able to extract an ordinary differential equation such that every solution of the DAE is a solution of this ODE. In [23] it has been shown that if both $k$ and $m$ are well-defined we have $u_{m}=0$ (i.e. no undetermined solution components) and $k=0$ if $m=0$ and $a_{0}=0$ or $k=m+1$ otherwise.

The finite sequences $r_{i}, a_{i}, s_{i}, i \in\{0, \ldots, m\}$ (recall that $d_{i}, u_{i}$ are not independent of these) are now sufficient to describe the possible existence and uniqueness results for $(1)$, see [23].

THEOREM 1.2. Let $m$ from (11) be well-defined for the DAE (1) and let $(E(t), A(t))$ be smooth matrix functions. Let $r_{i}, a_{i}, s_{i}, d_{i}, u_{i}, i \in\{0, \ldots, m\}$ be the related characteristic values as above. Furthermore let

$$
\left.\left(E_{i}(t), A_{i}(t)\right)=\left(\left[\begin{array}{ccccc}
I_{s_{i}} & 0 & 0 & 0 & 0 \\
0 & I_{d_{i}} & 0 & 0 & 0 \\
0 & 0 & 0 & 0 & 0 \\
0 & 0 & 0 & 0 & 0 \\
0 & 0 & 0 & 0 & 0
\end{array}\right],\left[\begin{array}{ccccc}
0 & A_{12}^{(i)}(t) & 0 & A_{14}^{(i)}(t) & A_{15}^{(i)}(t) \\
0 & 0 & 0 & A_{24}^{(i)}(t) & A_{25}^{(i)}(t) \\
0 & 0 & I_{a_{i}} & 0 & 0 \\
I_{s_{i}} & 0 & 0 & 0 & 0 \\
0 & 0 & 0 & 0 & 0
\end{array}\right]\right)\right) \begin{gathered}
s_{i} \\
d_{i} \\
a_{i} \\
s_{i} \\
u_{i}
\end{gathered} .
$$


and define

$$
\begin{aligned}
& \text { (a) } b_{0}=a_{0}, \quad b_{i}=\operatorname{rank}\left(\left[\begin{array}{ll}
A_{14}^{(i-1)}(t) & A_{15}^{(i-1)}(t)
\end{array}\right]\right), \\
& \text { (b) } c_{0}=a_{0}+s_{0}, \quad c_{i}=\operatorname{rank}\left(\left[\begin{array}{lll}
A_{12}^{(i-1)}(t) & A_{14}^{(i-1)}(t) & A_{15}^{(i-1)}(t)
\end{array}\right]\right) \text {, } \\
& \text { (c) } w_{0}=u_{0}, \quad w_{i}=u_{i}-u_{i-1}, \quad i=1, \ldots, m \text {. }
\end{aligned}
$$

Then we have

$\begin{array}{lll}\text { (a) } & c_{i}=b_{i}+s_{i}, & i=0, \ldots, m \\ \text { (b) } & w_{i}=s_{i-1}-c_{i}, & i=1, \ldots, m\end{array}$

and $(E(t), A(t))$ is equivalent to the pair of matrix functions (without arguments)

$$
\left(\left[\begin{array}{c|ccc|cccc}
I & 0 & \ldots & 0 & 0 & * & \ldots & * \\
\hline 0 & 0 & \ldots & 0 & 0 & F_{m} & & * \\
\vdots & \vdots & & \vdots & & \ddots & \ddots & \\
\vdots & \vdots & & \vdots & & & \ddots & F_{1} \\
0 & 0 & \ldots & 0 & & & & 0 \\
\hline 0 & 0 & \ldots & 0 & 0 & G_{m} & & * \\
\vdots & \vdots & & \vdots & & \ddots & \ddots & \\
\vdots & \vdots & & \vdots & & & \ddots & G_{1} \\
0 & 0 & \ldots & 0 & & & & 0
\end{array}\right],\left[\begin{array}{c|ccc|cccc}
* & * & \ldots & * & 0 & \ldots & \ldots & 0 \\
\hline 0 & 0 & \ldots & 0 & 0 & \ldots & \ldots & 0 \\
\vdots & \vdots & & \vdots & \vdots & & & \vdots \\
\vdots & \vdots & & \vdots & \vdots & & & \vdots \\
0 & 0 & \ldots & 0 & 0 & \ldots & \ldots & 0 \\
\hline 0 & 0 & \ldots & 0 & I & & & \\
\vdots & \vdots & & \vdots & & \ddots & & \\
\vdots & \vdots & & \vdots & & & \ddots & \\
0 & 0 & \ldots & 0 & & & & I
\end{array}\right]\right)\left[\begin{array}{l}
d_{m} \\
w_{m} \\
\vdots \\
\vdots \\
w_{0} \\
c_{m} \\
\vdots \\
\vdots \\
c_{0}
\end{array}\right.
$$

where the diagonal blocks are square and

$$
\operatorname{rank}\left(\left[\begin{array}{c}
F_{i} \\
G_{i}
\end{array}\right]\right)=c_{i}+w_{i}=s_{i-1} \leq c_{i-1} .
$$

Using this canonical form the following existence and uniqueness result is obtained in [23].

THEOREM 1.3. Let $m$ from (11) be well-defined for the differential-algebraic equation (1) and let $f \in C^{m}\left([\underline{t}, \bar{t}], \mathbf{C}^{n}\right)$. Then the following holds:

i) Equation (1) is equivalent to a differential-algebraic equation of the form (with a notation adapted to a simplified block structure, i.e. different to (9), (10))

$$
\begin{array}{ll}
\text { (a) } & \dot{x}_{1}(t)=A_{13}(t) x_{3}(t)+f_{1}(t) \\
\text { (b) } & 0=x_{2}(t)+f_{2}(t) \\
\text { (c) } & 0=f_{3}(t)
\end{array}
$$

in the sense that their solutions are in one-to-one correspondence. The inhomogeneity is determined by $f^{(0)}, \ldots, f^{(m)}$. Furthermore $d_{m}, a_{m}, u_{m}$ are the number of differential, algebraic and undetermined components of the unknown $x$ in (a), (b), (c) respectively.

ii) Equation (1) is solvable if and only if the $u_{m}$ functional consistency conditions

$$
f_{3}(t) \equiv 0
$$

are satisfied.

iii) An initial condition (2) is consistent if and only if in addition the $a_{m}$ conditions

$$
x_{2}\left(t_{0}\right)=-f_{2}\left(t_{0}\right)
$$


hold.

iv) The initial value problem (1), (2) is uniquely solvable if again in addition we have

$$
u_{m}=0 .
$$

Otherwise, we can choose $x_{3} \in C^{1}\left([\underline{t}, \bar{t}], \mathbf{C}^{u_{m}}\right)$ arbitrarily.

These results are so far of a theoretical nature, neither the inductive procedure of reduction to the form (12) nor the canonical form (17) have been obtained in a way that is feasable for numerical computation. It is the aim of the present paper to yield numerical procedures for determining the invariants of a given DAE as well as a canonical form similar to (17) and appropriate discretizations of it.

In Section 2 we describe how the strangeness index $m$ and the other invariants can be obtained by a numerically stable procedure and how this information can be used to get a normal form similar to but not quite as reduced as (17) but with the same numerically relevant properties. It is clear that the numerical solution of a system in the canonical form (17) requires a regularization procedure to deal with the undetermined components. Here we use the notion regularization for any method that fixes an analytical or numerical solution when no unique solution exists, i.e. in the presence of undetermined solution components and possibly violated consistency conditions (18). There are several obvious ways to treat undetermined components, either one fixes them ad hoc during the procedure or one uses a least square approach to deal with the nonuniqueness. These issues will be discussed in Section 3. Apart from the undetermined components and consistency checks for the initial conditions and the components in (17c), a discretization of a system in a form similar to (17) is easily obtained from any standard discretization scheme for ordinary differential equations, e.g. $[18,19]$. We will describe a discretization that is based on backward difference formulas (BDF) applied to a suitable canonical form. We have implemented the numerical procedures in an experimental FORTRAN code and have applied it to several test examples. These results are presented in Section 4.

2. Computing the Strangeness Index and a Canonical Form. In this section we describe a procedure that can be used to determine the strangeness index $m$ and the other invariants from the system (1) and its derivatives. This procedure then allows us to compute a normal form, for which we can then apply standard methods of regularization and discretization. In view of Theorem 1.3 this computable normal form should exhibit the correct solution behaviour. In particular it should be equivalent in the sense of Definition 2 to (17) as basis of the existence and uniqueness result obtained there. The overall numerical procedure for solving (1), (2) we aim at will then consist of transforming (1) to a normal form with the same solutions as (1) such that standard discretization of this normal form reflects the characteristics of the given DAE. Essentially, these characteristics are given by the numbers $d_{m}, a_{m}$ and $u_{m}=n-d_{m}-a_{m}$ as occuring in Theorem 1.3.

In the following we assume that the strangeness index $m$ is well-defined in the interval in which we want to solve (1). This in particular means that none of the invariant quantities determined during the procedure changes its value, as required in (8). It is clear that in a numerical procedure we must try to identify points where such a change takes place.

In the sequel we denote by a superscript ${ }^{(i)}$ the $i$-th derivative and we define for sufficiently smooth $E(t), A(t)$ as in (1) and for $\ell=0, \ldots, m$ the matrix functions 
$M_{\ell}(t), N_{\ell}(t)$ and the vector functions $z_{\ell}(t), g_{\ell}(t)$ componentwise by

$$
\begin{aligned}
& \left(M_{\ell}\right)_{i, j}:=\left(\begin{array}{c}
i \\
j
\end{array}\right) E^{(i-j)}-\left(\begin{array}{c}
i \\
j+1
\end{array}\right) A^{(i-j-1)}, i, j=0, \ldots, \ell \\
& \left(N_{\ell}\right)_{i, j}:= \begin{cases}A^{(i)} & \text { for } i=0, \ldots, \ell, j=0 \\
0 & \text { otherwise. }\end{cases} \\
& \left(z_{\ell}\right)_{i}:=x^{(i)}, i=0, \ldots, \ell \\
& \left(g_{\ell}\right)_{i}:=f^{(i)}, i=0, \ldots, \ell
\end{aligned}
$$

such that (1) and its derivatives up to order $\ell$ can be written as

$$
M_{\ell}(t) \dot{z}_{\ell}(t)=N_{\ell}(t) z_{\ell}(t)+g_{\ell}(t) .
$$

Note that a similar equation was already introduced by Campbell in [3], see also [2].

We will show now that at a fixed point $t_{0} \in[\underline{t}, \bar{t}]$ the characteristic quantities corresponding to the large matrix pair $\left(M_{\ell}\left(t_{0}\right), N_{\ell}\left(t_{0}\right)\right)$ are invariant under equivalence transformations to the original pair $(E(t), A(t))$.

To do this we need the following identities for binomial coefficients, where we use the convention that $\left(\begin{array}{l}i \\ j\end{array}\right)=0$ for $i<j, j<0, j>i$.

LEMma 2.1. For all integers $i, j, k, l$ we have the identities:

$$
\begin{aligned}
& \text { (a) }\left(\begin{array}{c}
i \\
j
\end{array}\right)\left(\begin{array}{c}
i-j \\
k
\end{array}\right)\left(\begin{array}{c}
i-j-k \\
l
\end{array}\right)+\left(\begin{array}{c}
i \\
j+1
\end{array}\right)\left(\begin{array}{c}
i-j-1 \\
k
\end{array}\right)\left(\begin{array}{c}
i-j-k-1 \\
l
\end{array}\right)=\left(\begin{array}{c}
i \\
k
\end{array}\right)\left(\begin{array}{c}
i-k \\
l
\end{array}\right)\left(\begin{array}{c}
i-k-l+1 \\
j+1
\end{array}\right), \\
& \text { (b) }\left(\begin{array}{c}
i \\
j+1
\end{array}\right)\left(\begin{array}{c}
i-j-1 \\
k
\end{array}\right)\left(\begin{array}{c}
i-j-k-1 \\
l
\end{array}\right)=\left(\begin{array}{c}
i \\
k
\end{array}\right)\left(\begin{array}{c}
i-k \\
l
\end{array}\right)\left(\begin{array}{c}
i-k-l \\
j+1
\end{array}\right), \\
& \text { (c) }\left(\begin{array}{c}
i \\
k-1
\end{array}\right)\left(\begin{array}{c}
i-k+1 \\
l
\end{array}\right)+\left(\begin{array}{c}
i \\
k
\end{array}\right)\left(\begin{array}{c}
i-k \\
l-1
\end{array}\right)+\left(\begin{array}{c}
i \\
k
\end{array}\right)\left(\begin{array}{c}
i-k \\
l
\end{array}\right)=\left(\begin{array}{c}
i+1 \\
k
\end{array}\right)\left(\begin{array}{c}
i+1-k \\
l
\end{array}\right) .
\end{aligned}
$$

Proof. The proof follows by straightforward calculation.

The next Lemma gives a formula for the derivative of a product of three matrix functions.

LEMMA 2.2. Let $D(t)=A(t) B(t) C(t)$ be the product of three sufficiently smooth matrix valued functions of appropriate dimensions. Then

$$
D^{(i)}(t)=\sum_{j=0}^{i} \sum_{k=0}^{i-j}\left(\begin{array}{l}
i \\
j
\end{array}\right)\left(\begin{array}{c}
i-j \\
k
\end{array}\right) A^{(j)} B^{(k)} C^{(i-j-k)} .
$$

Proof. The proof follows immediately by induction using (23c).

Our next result relates equivalence transformations applied to $(E(t), A(t))$ to those applied to $\left(M_{\ell}\left(t_{0}\right), N_{\ell}\left(t_{0}\right)\right)$.

THEOREM 2.3. Let the pairs $(E(t), A(t))$ and $(\tilde{E}(t), \tilde{A}(t))$ be equivalent via the transformation

$$
\begin{aligned}
& \tilde{E}(t)=P(t) E(t) Q(t) \\
& \tilde{A}(t)=P(t) A(t) Q(t)-P(t) E(t) \dot{Q}(t)
\end{aligned}
$$

as in (3) and let $\left(M_{\ell}, N_{\ell}\right),\left(\tilde{M}_{\ell}, \tilde{N}_{\ell}\right)$ be the corresponding large pairs constructed as in (21). If we set

$$
\begin{aligned}
& \left(\Pi_{\ell}\right)_{i, j}:=\left(\begin{array}{c}
i \\
j
\end{array}\right) P^{(i-j)},\left(\Theta_{\ell}\right)_{i, j}:=\left(\begin{array}{c}
i+1 \\
j+1
\end{array}\right) Q^{(i-j)} \\
& \left(\Psi_{\ell}\right)_{i, j}:= \begin{cases}Q^{(i+1)} & \text { for } i=0, \ldots, \ell, j=0 \\
0 & \text { otherwise } \\
7\end{cases}
\end{aligned}
$$


then it follows for every fixed point $t_{0} \in[\underline{t}, \bar{t}]$ and for $\ell=0, \ldots, m$ that

$$
\left(\tilde{M}_{\ell}\left(t_{0}\right), \tilde{N}_{\ell}\left(t_{0}\right)\right)=\Pi_{\ell}\left(t_{0}\right)\left(M_{\ell}\left(t_{0}\right), N_{\ell}\left(t_{0}\right)\right)\left[\begin{array}{cc}
\Theta_{\ell}\left(t_{0}\right) & -\Psi_{\ell}\left(t_{0}\right) \\
0 & \Theta_{\ell}\left(t_{0}\right)
\end{array}\right],
$$

i.e. the corresponding matrix pairs are locally equivalent.

Proof. All matrices $M_{\ell}, N_{\ell}, \tilde{M}_{\ell}, \tilde{N}_{\ell}, \Pi_{\ell}, \Theta_{\ell}, \Psi_{\ell}$ are block lower triangular with the same block structure. Thus, it is sufficient to prove the result for $\ell=m$. Observe furthermore that $N_{\ell}, \tilde{N}_{\ell}, \Psi_{\ell}$ have nonzero blocks only in the first block column. From Lemma 2.2 we obtain

$$
\begin{gathered}
\tilde{E}^{(i)}=\sum_{k_{1}=0}^{i} \sum_{k_{2}=0}^{i-k_{1}}\left(\begin{array}{c}
i \\
k_{1}
\end{array}\right)\left(\begin{array}{c}
i-k_{1} \\
k_{2}
\end{array}\right) P^{\left(k_{1}\right)} E^{\left(k_{2}\right)} Q^{\left(i-k_{1}-k_{2}\right)} \\
\tilde{A}^{(i)}=\sum_{k_{1}=0}^{i} \sum_{k_{2}=0}^{i-k_{1}}\left[\left(\begin{array}{c}
i \\
k_{1}
\end{array}\right)\left(\begin{array}{c}
i-k_{1} \\
k_{2}
\end{array}\right) P^{\left(k_{1}\right)} A^{\left(k_{2}\right)} Q^{\left(i-k_{1}-k_{2}\right)}-\right. \\
\left.\quad-\left(\begin{array}{c}
i \\
k_{1}
\end{array}\right)\left(\begin{array}{c}
i-k_{1} \\
k_{2}
\end{array}\right) P^{\left(k_{1}\right)} E^{\left(k_{2}\right)} Q^{\left(i+1-k_{1}-k_{2}\right)}\right] .
\end{gathered}
$$

Leaving out the argument $t_{0}$ we obtain

$$
\begin{aligned}
& \left(\Pi_{m} M_{m} \Theta_{m}\right)_{i, j}= \\
& =\sum_{l_{1}=j}^{i} \sum_{l_{2}=j}^{l_{1}}\left(\Pi_{m}\right)_{i, l_{1}}\left(M_{m}\right)_{l_{1}, l_{2}}\left(\Theta_{m}\right)_{l_{2}, j}= \\
& =\sum_{l_{1}=j}^{i} \sum_{l_{2}=j}^{l_{1}}\left(\begin{array}{c}
i \\
l_{1}
\end{array}\right) P^{\left(i-l_{1}\right)}\left[\left(\begin{array}{l}
l_{1} \\
l_{2}
\end{array}\right) E^{\left(l_{1}-l_{2}\right)}-\left(\begin{array}{c}
l_{1} \\
l_{2}+1
\end{array}\right) A^{\left(l_{1}-l_{2}-1\right)}\right]\left(\begin{array}{c}
l_{2}+1 \\
j+1
\end{array}\right) Q^{\left(l_{2}-j\right)}
\end{aligned}
$$

by inserting the definitions. Shifting and inverting the summations and applying $(23 a, b)$ then leads to

$$
\begin{aligned}
& \left(\Pi_{m} M_{m} \Theta_{m}\right)_{i, j}= \\
& =\left(\begin{array}{c}
i \\
j
\end{array}\right) \sum_{k_{1}=0}^{i-j} \sum_{k_{2}=0}^{i-j-k_{1}}\left(\begin{array}{c}
i-j \\
k_{1}
\end{array}\right) P^{\left(k_{1}\right)}\left(\begin{array}{c}
i-j-k_{1} \\
k_{2}
\end{array}\right) E^{\left(k_{2}\right)} Q^{\left(i-j-k_{1}-k_{2}\right)} \\
& \quad-\left(\begin{array}{c}
i \\
j+1
\end{array}\right) \sum_{k_{1}=0}^{i-j-1} \sum_{k_{2}=0}^{i-j-1-k_{1}}\left[\left(\begin{array}{c}
i-j-1 \\
k_{1}
\end{array}\right) P^{\left(k_{1}\right)}\left(\begin{array}{c}
i-j-k_{1}-1 \\
k_{2}
\end{array}\right) A^{\left(k_{2}\right)} Q^{\left(i-j-1-k_{1}-k_{2}\right)}\right. \\
& \left.\quad \quad-\left(\begin{array}{c}
i-j-1 \\
k_{1}
\end{array}\right) P^{\left(k_{1}\right)}\left(\begin{array}{c}
i-j-1-k_{1} \\
k_{2}
\end{array}\right) E^{\left(k_{2}\right)} Q^{\left(i-j-k_{1}-k_{2}\right)}\right]= \\
& =\left(\begin{array}{c}
i \\
j
\end{array}\right) \tilde{E}^{(i-j)}-\left(\begin{array}{c}
i \\
j+1
\end{array}\right) \tilde{A}^{(i-j-1)}=\left(\tilde{M}_{m}\right)_{i, j} .
\end{aligned}
$$

In a similar way it follows that

$$
\begin{aligned}
& \left(\Pi_{m} N_{m} \Theta_{m}\right)_{i, 0}-\left(\Pi_{m} M_{m} \Psi_{m}\right)_{i, 0}= \\
& =\sum_{l_{1}=0}^{i}\left(\Pi_{m}\right)_{i, l_{1}}\left(N_{m}\right)_{l_{1}, 0}\left(\Theta_{m}\right)_{0,0} \\
& -\sum_{l_{1}=0}^{i} \sum_{l_{2}=0}^{l_{1}}\left(\Pi_{m}\right)_{i, l_{1}}\left(M_{m}\right)_{l_{1}, l_{2}}\left(\Psi_{m}\right)_{l_{2}, 0}= \\
& =\sum_{k_{1}=0}^{i}\left(\begin{array}{c}
i \\
k_{1}
\end{array}\right) P^{\left(k_{1}\right)} A^{\left(i-k_{1}\right)} Q^{(0)}+ \\
& +\sum_{k_{1}=0}^{i} \sum_{k_{2}=0}^{i-1-k_{1}}\left(\begin{array}{c}
i \\
k_{1}
\end{array}\right) P^{\left(k_{1}\right)}\left(\begin{array}{c}
i-k_{1} \\
k_{2}
\end{array}\right) A^{\left(k_{2}\right)} Q^{\left(i-k_{1}-k_{2}\right)} \\
& -\sum_{k_{1}=0}^{i} \sum_{k_{2}=0}^{i-k_{1}}\left(\begin{array}{c}
i \\
k_{1}
\end{array}\right) P^{\left(k_{1}\right)}\left(\begin{array}{c}
i-k_{1} \\
k_{2}
\end{array}\right) E^{\left(k_{2}\right)} Q^{\left(i-k_{1}-k_{2}+1\right)}= \\
& =\tilde{A}^{(i)}=\left(\tilde{N}_{m}\right)_{i, 0} \text {. }
\end{aligned}
$$

Thus we have shown that the characteristic quantities $\left(\tilde{r}_{\ell}, \tilde{a}_{\ell}, \tilde{s}_{\ell}, \tilde{d}_{\ell}, \tilde{u}_{\ell}\right)$ of the large pair $\left(M_{\ell}\left(t_{0}\right), N_{\ell}\left(t_{0}\right)\right)$ are well-defined for equivalent pairs of matrix functions and each $\ell \in \mathbf{N}_{0}$. Since these quantities are numerically computable via three rank decisions for each $\ell$, it remains the question, whether we can decide from these quantities on 
the size of $m$ and on the quantities $\left(r_{i}, a_{i}, s_{i}, d_{i}, u_{i}\right), i=1, \ldots, m$ of the original pair $(E(t), A(t))$ at the point $t=t_{0}$.

THEOREM 2.4. The characteristic quantities $\left(\tilde{r}_{\ell}, \tilde{a}_{\ell}, \tilde{s}_{\ell}, \tilde{d}_{\ell}, \tilde{u}_{\ell}\right)$ of $\left(M_{\ell}\left(t_{0}\right), N_{\ell}\left(t_{0}\right)\right)$ and $\left(r_{i}, a_{i}, s_{i}, d_{i}, u_{i}\right)$ of $(E(t), A(t))$ are related by the following formulas:

$$
\begin{array}{rlrl}
\tilde{r}_{\ell}=(\ell+1) n-\sum_{i=0}^{\ell} c_{i}-\sum_{i=0}^{\ell} u_{i}, & \tilde{r}_{m}=(m+1) n-a_{m}-\sum_{i=0}^{m} u_{i} \\
\tilde{a}_{\ell}=s_{\ell-1}-w_{\ell}-s_{\ell}=c_{\ell}-s_{\ell}, & \tilde{a}_{m}=c_{m} \\
\tilde{s}_{\ell}=s_{\ell}+\sum_{i=0}^{\ell-1} c_{i}, & \tilde{s}_{m}=\sum_{i=0}^{m-1} c_{i}=a_{m}-c_{m} \\
\tilde{d}_{\ell}=\tilde{r}_{\ell}-s_{\ell}=(\ell+1) n-c_{\ell}-\sum_{i=0}^{\ell} u_{i}, & \tilde{d}_{m}=(m+1) n-c_{m}-\sum_{i=0}^{m} u_{i} \\
\tilde{u}_{\ell}=(\ell+1) n-\tilde{r}_{\ell}-\tilde{a}_{\ell}-\tilde{s}_{\ell}=\sum_{i=0}^{\ell} u_{i}, & & \tilde{u}_{m}=\sum_{i=0}^{m} u_{i} \\
\quad & \quad=0, \ldots, m &
\end{array}
$$

Proof. By Theorem 2.3 we may assume w.l.o.g. that the pair $(E(t), A(t))$ is already given in normal form (15). For fixed $t_{0}$ we can apply the local equivalence transformation $(27)$ to $\left(M_{\ell}, N_{\ell}\right)$. First, we form the pair $\left(\breve{M}_{\ell}, \breve{N}_{\ell}\right)$ from the blocks

$$
\left(\breve{M}_{\ell}\right)_{i, j}:=\left(M_{\ell}\right)_{\ell-i, \ell-j},\left(\breve{N}_{\ell}\right)_{i, j}:=\left(N_{\ell}\right)_{\ell-i, \ell-j}
$$

and obtain

$$
\breve{M}_{\ell}=\left[\begin{array}{cccccc}
T_{0,0} & T_{0,1} & T_{0,2} & \ldots & T_{0, \ell-1} & T_{0, \ell} \\
& T_{1,1} & T_{1,2} & \ldots & T_{1, \ell-1} & T_{1, \ell} \\
& & T_{2,2} & \ldots & T_{2, \ell-1} & T_{2, \ell} \\
& & & \ddots & \vdots & \vdots \\
& & & & T_{\ell-1, \ell-1} & T_{\ell-1, \ell} \\
& & & & & T_{\ell, \ell}
\end{array}\right] \begin{gathered}
0 \\
\ell \\
\end{gathered}
$$

and

$$
\breve{N}_{\ell}=\left[\begin{array}{cccc}
0 & \ldots & 0 & S_{0, \ell} \\
0 & \ldots & 0 & S_{1, \ell} \\
\vdots & & \vdots & \vdots \\
0 & \ldots & 0 & S_{\ell, \ell}
\end{array}\right] \begin{gathered}
0 \\
1 \\
\vdots \\
\ell
\end{gathered}
$$

where each of the blocks $T_{i, i}, i=0, \ldots, \ell$ has the form

$$
\left[\begin{array}{c|ccc|cccc}
I & 0 & \ldots & 0 & 0 & * & \ldots & * \\
\hline 0 & 0 & \ldots & 0 & 0 & F_{m} & * & * \\
\vdots & \vdots & & \vdots & & \ddots & \ddots & * \\
\vdots & \vdots & & \vdots & & & \ddots & F_{1} \\
0 & 0 & \ldots & 0 & & & & 0 \\
\hline 0 & 0 & \ldots & 0 & 0 & G_{m} & & * \\
\vdots & \vdots & & \vdots & & \ddots & \ddots & \\
\vdots & \vdots & & \vdots & & & \ddots & G_{1} \\
0 & 0 & \ldots & 0 & & & & 0
\end{array}\right],
$$


each of the blocks $T_{i, i+1}, i=0, \ldots, \ell-1$ has the form

$$
\left[\begin{array}{c|ccc|cccc}
* & * & \ldots & * & 0 & * & \ldots & * \\
\hline 0 & 0 & \ldots & 0 & 0 & * & \ldots & * \\
\vdots & \vdots & & \vdots & \vdots & & \ddots & \vdots \\
\vdots & \vdots & & \vdots & \vdots & & & * \\
0 & 0 & \ldots & 0 & 0 & \ldots & \ldots & 0 \\
\hline 0 & 0 & \ldots & 0 & -I & * & \ldots & * \\
\vdots & \vdots & & \vdots & & \ddots & \ddots & \vdots \\
\vdots & \vdots & & \vdots & & & \ddots & * \\
0 & 0 & \ldots & 0 & & & & -I
\end{array}\right]
$$

and each of the other blocks in the upper triangular part of $\breve{M}_{\ell}$ has the form

$$
\left[\begin{array}{c|ccc|cccc}
* & * & \ldots & * & 0 & * & \ldots & * \\
\hline 0 & 0 & \ldots & 0 & 0 & * & \ldots & * \\
\vdots & \vdots & & \vdots & \vdots & & \ddots & \vdots \\
\vdots & \vdots & & \vdots & \vdots & & & * \\
0 & 0 & \ldots & 0 & 0 & \ldots & \ldots & 0 \\
\hline 0 & 0 & \ldots & 0 & 0 & * & \ldots & * \\
\vdots & \vdots & & \vdots & & \ddots & \ddots & \vdots \\
\vdots & \vdots & & \vdots & & & \ddots & * \\
0 & 0 & \ldots & 0 & & & & 0
\end{array}\right] .
$$

In $\breve{N}_{\ell}$ each of the blocks $S_{i, \ell}, i=0, \ldots, \ell-1$ has the form

$$
\left[\begin{array}{c|ccc|ccc}
* & * & \ldots & * & 0 & \ldots & 0 \\
\hline 0 & 0 & \ldots & 0 & 0 & \ldots & 0 \\
\vdots & \vdots & & \vdots & \vdots & & 0 \\
0 & 0 & \ldots & 0 & 0 & \ldots & 0 \\
\hline 0 & 0 & \ldots & 0 & 0 & \ldots & 0 \\
\vdots & \vdots & & \vdots & \vdots & & \vdots \\
0 & 0 & \ldots & 0 & 0 & \ldots & 0
\end{array}\right]
$$

and $S_{\ell, \ell}$ has the form

$$
\left[\begin{array}{c|ccc|ccc}
* & * & \ldots & * & 0 & \ldots & 0 \\
\hline 0 & 0 & \ldots & 0 & 0 & \ldots & 0 \\
\vdots & \vdots & & \vdots & \vdots & & 0 \\
0 & 0 & \ldots & 0 & 0 & \ldots & 0 \\
\hline 0 & 0 & \ldots & 0 & I & & \\
\vdots & \vdots & & \vdots & & \ddots & \\
0 & 0 & \ldots & 0 & & & I
\end{array}\right]
$$

We can simplify the matrix $\breve{M}_{\ell}$ by using block Gaussian elimination with the identity blocks in the following way. We use the identity matrix in the upper left corner of 
each block $T_{i, i}$ to eliminate all other block entries in the corresponding block row of $\breve{M}_{\ell}$ by a corresponding block Gauss-elimination step applied to the right. Then one can eliminate with the lower right identity matrix in the $(0,1)$-block all other blocks in the corresponding block column, then with the lower two identity blocks in the $(1,2)$-block all other blocks in the corresponding block columns and inductively with the lower $\ell$ identity blocks of the $(\ell-1, \ell$ )-block all other blocks in the last $\ell$ block columns. Let $\left(\hat{M}_{\ell}, \hat{N}_{\ell}\right)$ be the pair that we obtain at the end of this process. It has the same coarse block structure $(30),(31)$ as $\left(\breve{M}_{\ell}, \breve{N}_{\ell}\right)$, i.e. none of the structural zero blocks in $\breve{N}_{\ell}$ is destroyed. We denote the blocks of $\hat{M}_{\ell}, \hat{N}_{\ell}$ by $\hat{T}_{i, j}, \hat{S}_{i, j}$, respectively.

The rank defect that occurs in the nilpotent part of the diagonal blocks $\hat{T}_{i, i}, i=$ $1, \ldots, \ell-1$ is for the $c_{i}$ blocks (see (15)) compensated by the identity blocks in the blocks $\hat{T}_{i, i+1}, i=1, \ldots, \ell-1$. Thus we obtain

$$
\begin{aligned}
\tilde{r}_{\ell}= & \left(n-w_{0}\right)+\left(n-w_{0}-w_{1}\right)+\left(n-w_{0}-w_{1}-w_{2}\right)+\ldots+ \\
& \quad+\left(n-w_{0}-\ldots-w_{\ell-1}\right)+\left(n-w_{0}-\ldots-w_{\ell}-c_{0}-\ldots-c_{\ell}\right)= \\
= & (\ell+1) n-\sum_{i=0}^{\ell} u_{i}-\sum_{i=0}^{\ell} c_{i},
\end{aligned}
$$

where we have used (13c).

In order to determine the other invariants of $\hat{M}_{\ell}, \hat{N}_{\ell}$ as in Theorem 1.1, we need $Z_{\ell}^{*} \hat{N}_{\ell} T_{\ell}$ and $Z_{\ell}^{*} \hat{N}_{\ell} T_{\ell}^{\prime}$, where the columns of $Z_{\ell}$ span the corange of $\hat{M}_{\ell}$ and the columns of $T_{\ell}, T_{\ell}^{\prime}$ span the kernel and cokernel of $\hat{M}_{\ell}$, respectively.

Since $\hat{N}_{\ell}$ has nonvanishing block entries only in the last block column and $Z_{\ell}$ has a zero block in its upper left corner of each block (due to the identity in the corresponding block of $\hat{M}_{\ell}$ ), the only relevant block in $Z_{\ell}^{*} \hat{N}_{\ell}$ that contributes to the desired ranks is the one in the last one, which has the form

$$
\begin{gathered}
w_{\ell} \\
\vdots \\
w_{0} \\
c_{\ell} \\
\vdots \\
c_{0}
\end{gathered}\left[\begin{array}{l|cccc|ccc}
0 & 0 & 0 & \ldots & 0 & 0 & \ldots & 0 \\
\hline & 0 & 0 & \ldots & 0 & 0 & \ldots & 0 \\
& \vdots & \vdots & & \vdots & \vdots & & \vdots \\
& 0 & 0 & \ldots & 0 & 0 & \ldots & 0 \\
\hline & & & & & I & & \\
& & & & & & \ddots & \\
& & & & & & I
\end{array}\right] .
$$

We obtain the kernel of $\hat{M}_{\ell}$ from the zero columns and the right nullspaces of the blocks $F_{i}, G_{i}$. Set

$$
U_{i}:=\left[\begin{array}{c}
F_{i} \\
G_{i}
\end{array}\right]
$$

and let the columns of $K_{i}, K_{i}^{\prime}$ form orthonormal bases of kernel $U_{i}$, cokernel $U_{i}$, respectively. By (16) we have that $U_{i}$ is a $\left(c_{i}+w_{i}, c_{i-1}\right)$-matrix of full row rank, thus $\operatorname{rank}\left(K_{i}\right)=c_{i-1}-c_{i}-w_{i}$.

Observe that due to the full row rank of $U_{i}$ part of the identity blocks in $\hat{T}_{i, i+1}$ can be eliminated by the full rank part of $\hat{T}_{i, i}$.

Hence we obtain that

$$
\operatorname{dim}\left(\operatorname{kernel}\left(\hat{M}_{\ell}\right)\right)=\sum_{i=0}^{\ell}\left(\sum_{j=i+1}^{m}\left(c_{j-1}-c_{j}-w_{j}\right)+c_{m}+\sum_{j=0}^{m} w_{j}\right)=\sum_{i=0}^{\ell}\left(c_{i}+u_{i}\right) .
$$


Now due to the form of $Z_{\ell} \hat{N}_{\ell}$ the only block of $\hat{T}_{\ell}$ that is relevant is the last one and it has the form

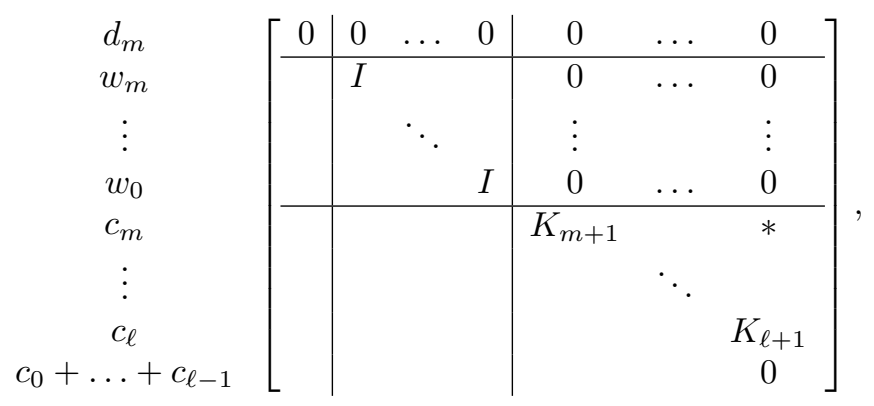

with $K_{m+1}=I$.

For $\hat{T}_{\ell}^{\prime}$, we have the last block

$d_{m}$
$w_{m}$
$\vdots$
$w_{0}$
$c_{\ell}+\ldots+c_{m}$
$c_{\ell-1}$
$\vdots$
$c_{0}$$\left[\begin{array}{ccc|c|ccc}0 & \ldots & 0 & I & 0 & \ldots & 0 \\ \hline 0 & \ldots & 0 & 0 & 0 & \ldots & 0 \\ \vdots & & \vdots & \vdots & \vdots & & \vdots \\ 0 & \ldots & 0 & 0 & 0 & \ldots & 0 \\ \hline & & & 0 & 0 & \ldots & 0 \\ & & & & & & \\ & & & & & & \\ & & & & & & \\ \prime\end{array}\right.$

Putting these results together we obtain as in Theorem 1.1,

$$
\tilde{a}_{\ell}=\operatorname{rank}\left(Z_{\ell}^{*} \hat{N}_{\ell} \hat{T}_{\ell}\right)=\operatorname{rank}\left(K_{\ell+1}\right)=c_{\ell}-c_{\ell+1}-w_{\ell+1}=s_{\ell-1}-w_{\ell}-s_{\ell}
$$

and

$$
\tilde{s}_{\ell}=c_{0}+\ldots+c_{\ell-1}+\operatorname{rank}\left(K_{\ell+1}^{\prime}\right)=c_{0}+\ldots+c_{\ell-1}+c_{\ell+1}+w_{\ell+1}=s_{\ell}+\sum_{i=0}^{\ell-1} c_{i} .
$$

Thus we have finished the proof. $\quad \square$

As a corollary we obtain recursive formulas for the characteristic values $\left(r_{i}, a_{i}, s_{i}, d_{i}, u_{i}\right)$ of the original pair $(E(t), A(t))$ :

COROLlaRY 2.5. The following recursive formulas hold:

$$
\begin{aligned}
r_{0} & =\tilde{r}_{0}, & r_{i} & =r_{i-1}-s_{i-1} \\
a_{0} & =\tilde{a}_{0}, & s_{i} & =\tilde{s}_{i}-v_{i-1} \\
s_{0} & =\tilde{s}_{0}, & w_{i} & =s_{i-1}-s_{i}-\tilde{a}_{i} \\
d_{0} & =\tilde{d}_{0}, & u_{i} & =w_{i}-u_{i-1} \\
u_{0} & =\tilde{u}_{0}, & & a_{i}=n-r_{i}-s_{i}-u_{i} \\
w_{0} & =u_{0}, & & d_{i}=r_{i}-s_{i} \\
c_{0} & =a_{0}+s_{0}, & c_{i} & =s_{i-1}-w_{i} \\
b_{0} & =a_{0}, & b_{i} & =c_{i}-s_{i} \\
v_{0} & =s_{0}, & v_{i} & =v_{i-1}+c_{i}
\end{aligned}
$$


Proof. The values of $r_{i}$ follow from the construction of the sequence and the values of $s_{i}$ and $w_{i}$ follow directly from the formulas for $\tilde{s}_{i}$ and $\tilde{a}_{i}$. The other formulas are then clear.

These recursive formulas now give a numerically computable method for the determination of the strangeness index $m$ since by knowledge of the sequence $s_{i}$ the quantity $m$ follows directly from its definition (11).

The next task is now the extraction of a strangeness free matrix pair $(\hat{E}(t), \hat{A}(t))$ and the corresponding DAE with local characteristic quantities $\hat{r}=d_{m}, \hat{a}=a_{m}, \hat{s}=0$ the latter having the same solution structure as the original DAE (1). For reasons of numerical stability, the extraction procedure to be described below will be exclusively based on the use of unitary projectors in the form of matrices with orthonormal columns.

In the following we assume that all the characteristic quantities of $\left(M_{\ell}(t), N_{\ell}(t)\right), \ell=0, \ldots, m$ are independent of $t$. Let $(\tilde{E}(t), \tilde{A}(t))$ be the normal form (15) of $(E(t), A(t))$ and let $\left(\tilde{M}_{\ell}(t), \tilde{N}_{\ell}(t)\right), \quad \ell=0, \ldots, m$ be the correponding large pairs. In the following we omit the index $m$, i.e. $M_{m}=: M, N_{m}=: N$, $\tilde{M}_{m}=: \tilde{M}, \tilde{N}_{m}=: \tilde{N}$.

By the proof of Theorem 2.4 in connection with $\tilde{a}_{m}+\tilde{s}_{m}=a_{m}$, we observe that there exists a smooth matrix valued function $\tilde{Z}_{2}(t)$ of size $\left((m+1) n, a_{m}\right)$ with orthonormal columns, such that

$$
\tilde{Z}_{2}(t)^{*} \tilde{M}(t)=0
$$

and

$$
\operatorname{rank}\left(\tilde{Z}_{2}(t)^{*} \tilde{N}(t)\left[\begin{array}{c}
I_{n} \\
0 \\
\vdots \\
0
\end{array}\right]\right)=\operatorname{rank}\left(\left[\begin{array}{c}
0 \\
0 \\
I_{a_{m}}
\end{array}\right]^{*}\left[\begin{array}{ccc}
* & * & 0 \\
0 & 0 & 0 \\
0 & 0 & I_{a_{m}}
\end{array}\right]\right)=a_{m}
$$

If we now set

$$
\tilde{T}_{2}(t):=\left[\begin{array}{cc}
I_{d_{m}} & 0 \\
0 & I_{u_{m}} \\
0 & 0
\end{array}\right]
$$

we then have

$$
\tilde{Z}_{2}(t)^{*} \tilde{N}(t)\left[\begin{array}{c}
I_{n} \\
0 \\
\vdots \\
0
\end{array}\right] \tilde{T}_{2}(t)=0
$$

and

$$
\operatorname{rank}\left(\tilde{E}(t) \tilde{T}_{2}(t)\right)=\operatorname{rank}\left(\left[\begin{array}{ccc}
I_{d_{m}} & 0 & * \\
0 & 0 & F(t) \\
0 & 0 & G(t)
\end{array}\right]\left[\begin{array}{cc}
I_{d_{m}} & 0 \\
0 & I_{u_{m}} \\
0 & 0
\end{array}\right]\right)=d_{m}
$$

¿From

$$
\begin{aligned}
& \tilde{M}(t)=\Pi(t) M(t) \Theta(t), \\
& \tilde{N}(t)=\Pi(t) N(t) \Theta(t)-\Pi(t) M(t) \Psi(t)
\end{aligned}
$$


it follows that

$$
\tilde{Z}_{2}(t)^{*} \tilde{M}(t)=\tilde{Z}_{2}(t)^{*} \Pi(t) M(t) \Theta(t)=0
$$

and due to the special structure of $N$

$$
\begin{aligned}
a_{m} & =\operatorname{rank}\left(\tilde{Z}_{2}(t)^{*} \tilde{N}(t)\left[\begin{array}{cccc}
I_{n} & 0 & \ldots & 0
\end{array}\right]^{*}\right)= \\
& =\operatorname{rank}\left(\tilde{Z}_{2}(t)^{*} \Pi(t) N(t) \Theta(t)\left[\begin{array}{llll}
I_{n} & 0 & \ldots & 0
\end{array}\right]^{*}\right)= \\
& =\operatorname{rank}\left(\tilde{Z}_{2}(t)^{*} \Pi(t) N(t)\left[\begin{array}{lllll}
Q^{(0)}(t) & * & \ldots & *
\end{array}\right]^{*}\right)= \\
& =\operatorname{rank}\left(\tilde{Z}_{2}(t)^{*} \Pi(t) N(t)\left[\begin{array}{lllll}
Q^{(0)}(t) & 0 & \ldots & 0
\end{array}\right]^{*}\right)= \\
& =\operatorname{rank}\left(\tilde{Z}_{2}(t)^{*} \Pi(t) N(t)\left[\begin{array}{llll}
I_{n} & 0 & \ldots & 0
\end{array}\right]^{*} Q^{(0)}(t)\right),
\end{aligned}
$$

i.e. there exists a smooth matrix valued function $Z_{2}(t)$, which we may assume w.l.o.g. to have orthonormal columns (by orthonormalizing the columns of $\Pi(t)^{*} \tilde{Z}_{2}(t)$ ), such that

$$
Z_{2}(t)^{*} M(t)=0, \quad \operatorname{rank}\left(Z_{2}(t)^{*} N(t)\left[\begin{array}{c}
I_{n} \\
0 \\
\vdots \\
0
\end{array}\right]\right)=a_{m}
$$

¿From

$$
\begin{aligned}
0 & =\tilde{Z}_{2}(t)^{*} \tilde{N}(t)\left[\begin{array}{cccc}
I_{n} & 0 & \ldots & 0
\end{array}\right]^{*} \tilde{T}_{2}(t)= \\
& =\tilde{Z}_{2}(t)^{*} \Pi(t) N(t) \Theta(t)\left[\begin{array}{cccc}
I_{n} & 0 & \ldots & 0
\end{array}\right]^{*} \tilde{T}_{2}(t)= \\
& =\tilde{Z}_{2}(t)^{*} \Pi(t) N(t)\left[\begin{array}{llll}
I_{n} & 0 & \ldots & 0
\end{array}\right]^{*} Q^{(0)} \tilde{T}_{2}(t)
\end{aligned}
$$

and

$$
\begin{aligned}
d_{m} & =\operatorname{rank}\left(\tilde{E}(t) \tilde{T}_{2}(t)\right)= \\
& =\operatorname{rank}\left(P^{(0)}(t) E(t) Q^{(0)}(t) \tilde{T}_{2}(t)\right)= \\
& =\operatorname{rank}\left(E(t) Q^{(0)}(t) \tilde{T}_{2}(t)\right),
\end{aligned}
$$

it follows that there exists a smooth matrix valued function $T_{2}(t)$ of size $\left(n, d_{m}+u_{m}\right)$, w.l.o.g. with orthonormal columns, such that

$$
Z_{2}(t)^{*} N(t)\left[\begin{array}{c}
I_{n} \\
0 \\
\vdots \\
0
\end{array}\right] T_{2}(t)=0, \quad \operatorname{rank}\left(E(t) T_{2}(t)\right)=d_{m}
$$

Thus, there exists a smooth matrix function $Z_{1}(t)$ of size $\left(n, d_{m}\right)$, also with orthonormal columns, such that

$$
\operatorname{rank}\left(Z_{1}(t)^{*} E(t) T_{2}(t)\right)=d_{m}
$$

Now instead of $Z_{1}(t)$ and $Z_{2}(t)$ we are only able to compute $Z_{1}(t) U_{1}(t)$ and $Z_{2}(t) U_{2}(t)$, where $U_{1}(t), U_{2}(t)$ are unitary but not necessarily smooth. The computable matrix pair that we obtain is then

$$
(\hat{E}(t), \hat{A}(t))=\left(\left[\begin{array}{c}
\hat{E}_{1}(t) \\
0 \\
0
\end{array}\right],\left[\begin{array}{c}
\hat{A}_{1}(t) \\
\hat{A}_{2}(t) \\
0
\end{array}\right]\right)
$$


where

(40) $\hat{E}_{1}(t)=U_{1}(t)^{*} Z_{1}(t)^{*} E(t)$,

$E_{1}(t)=U_{1}(t)^{*} Z_{1}(t)^{*} E(t)$,
$\hat{A}_{1}(t)=U_{1}(t)^{*} Z_{1}(t)^{*} A(t), \quad \hat{A}_{2}(t)=U_{2}(t)^{*} Z_{2}(t)^{*} N(t)\left[\begin{array}{llll}I_{n} & 0 & \ldots & 0\end{array}\right]^{*}$

Observe that $(\hat{E}(t), \hat{A}(t))$ may be non-smooth but that the product $\operatorname{diag}\left(U_{1}(t), U_{2}(t), I_{u_{m}}\right)(\hat{E}(t), \hat{A}(t))$ is smooth. The numerical method that we describe below is invariant under unitary transformations from the left, thus, since $\operatorname{diag}\left(U_{1}(t), U_{2}(t), I_{u_{m}}\right)$ is unitary, we can assume w.l.o.g. that $(\hat{E}(t), \hat{A}(t))$ is smooth.

We now have the following result for the invariants of $(\hat{E}(t), \hat{A}(t))$.

THEOREM 2.6. The characteristic quantities of the pair $(\hat{E}(t), \hat{A}(t))$ are uniformly given by

$$
(\hat{r}, \hat{a}, \hat{s}, \hat{d}, \hat{u})=\left(d_{m}, a_{m}, 0, d_{m}, u_{m}\right)
$$

Proof. To determine the characteristic quantities of $(\hat{E}(t), \hat{A}(t))$ we multiply from the left by the nonsingular matrix $\left[T_{1}^{\prime}, T_{2}^{\prime}, T_{3}\right]$, where $T_{2}=\left[T_{1}^{\prime}, T_{3}\right]$ in the above notation. The blocks $T_{1}^{\prime}$ and $T_{2}^{\prime}$ multiply into the cokernel of $\hat{E}_{1}$ and $\hat{A}_{2}$, respectively, thus we have that $\hat{E}_{1} T_{1}^{\prime}$ and $\hat{A}_{2} T_{2}^{\prime}$ are nonsingular. We get the following local equivalences (omitting arguments):

$$
\begin{aligned}
& (\hat{E}, \hat{A})=\left(\left[\begin{array}{c}
\hat{E}_{1} \\
0 \\
0
\end{array}\right],\left[\begin{array}{c}
\hat{A}_{1} \\
\hat{A}_{2} \\
0
\end{array}\right]\right) \rightarrow \\
& \stackrel{\mathrm{Q}}{\rightarrow}\left(\left[\begin{array}{ccc}
\hat{E}_{1} T_{1}^{\prime} & \hat{E}_{1} T_{2}^{\prime} & \hat{E}_{1} T_{3} \\
0 & 0 & 0 \\
0 & 0 & 0
\end{array}\right],\left[\begin{array}{ccc}
\hat{A}_{1} T_{1}^{\prime} & \hat{A}_{1} T_{2}^{\prime} & \hat{A}_{1} T_{3} \\
0 & \hat{A}_{2} T_{2}^{\prime} & 0 \\
0 & 0 & 0
\end{array}\right]\right) \rightarrow \\
& \stackrel{\mathrm{Q}}{\rightarrow}\left(\left[\begin{array}{ccc}
\hat{E}_{1} T_{1}^{\prime} & 0 & 0 \\
0 & 0 & 0 \\
0 & 0 & 0
\end{array}\right],\left[\begin{array}{ccc}
* & * & * \\
0 & \hat{A}_{2} T_{2}^{\prime} & 0 \\
0 & 0 & 0
\end{array}\right]\right) \rightarrow \\
& \stackrel{\mathrm{P}}{\rightarrow}\left(\left[\begin{array}{ccc}
I_{d_{m}} & 0 & 0 \\
0 & 0 & 0 \\
0 & 0 & 0 \\
I_{d_{m}} & 0 & 0 \\
0 & 0 & 0 \\
0 & 0 & 0
\end{array}\right],\left[\begin{array}{ccc}
* & * & * \\
0 & I_{a_{m}} & 0 \\
0 & 0 & 0 \\
0 & 0 & 0 \\
0 & I_{a_{m}} & 0 \\
0 & 0 & 0
\end{array}\right]\right) \rightarrow
\end{aligned}
$$

and we can read off $\hat{r}=d_{m}, \hat{a}=a_{m}, \hat{s}=0$.

Summarizing this section, we have introduced a numerically computable way to determine the local characterizing quantities of $(E(t), A(t))$ as well as a normal form which has the same solution structure as the original DAE but is now strangeness free. We can now discuss the regularization and discretization of this normal form.

3. Regularization and Discretization of a DAE in Normal Form. In this section we describe a regularized discretization for the linear DAE with variable coefficients in normal form

$$
\hat{E}(t) \dot{x}(t)=\hat{A}(t) x(t)+\hat{f}(t)
$$


where $(\hat{E}, \hat{A})$ are as in $(39)$ and

$$
\hat{f}(t)=\left[\begin{array}{c}
U_{1}(t)^{*} Z_{1}(t)^{*} g(t) \\
U_{2}(t)^{*} Z_{2}(t)^{*} g(t) \\
0
\end{array}\right]
$$

with $g:=g_{m}$ from (21). The discretization is based on backward-difference formulas (BDF), see e.g. [18]. The general $k$-step BDF method for $\dot{x}=f(t, x)$ is given by

$$
\sum_{i=0}^{k} \alpha_{i} x_{\ell-i}=h f\left(t_{\ell}, x_{\ell}\right),
$$

where the coefficients are given by the table

$$
\begin{array}{c|ccccccc}
\alpha_{i} & i=0 & i=1 & i=2 & i=3 & i=4 & i=5 & i=6 \\
\hline k=1 & 1 & -1 & & & & & \\
k=2 & \frac{3}{2} & -2 & \frac{1}{2} & & & & \\
k=3 & \frac{11}{6} & -3 & \frac{3}{2} & -\frac{1}{3} & & & \\
k=4 & \frac{25}{12} & -4 & 3 & -\frac{4}{3} & \frac{1}{4} & & \\
k=5 & \frac{137}{60} & -5 & 5 & -\frac{10}{3} & \frac{5}{4} & -\frac{1}{5} & \\
k=6 & \frac{147}{60} & -6 & \frac{15}{2} & -\frac{20}{3} & \frac{15}{4} & -\frac{6}{5} & \frac{1}{6}
\end{array}
$$

With this scheme we discretize the DAE (43) as

$$
\hat{E}\left(t_{\ell}\right) \sum_{i=0}^{k} \alpha_{i} x_{\ell-i}=h\left[\hat{A}\left(t_{\ell}\right) x_{\ell}+\hat{f}\left(t_{\ell}\right)\right]
$$

or

$$
\left[\alpha_{0} \hat{E}\left(t_{\ell}\right)-h \hat{A}\left(t_{\ell}\right)\right] x_{\ell}=h \hat{f}\left(t_{\ell}\right)-\hat{E}\left(t_{\ell}\right) \sum_{i=1}^{k} \alpha_{i} x_{\ell-i}
$$

By construction we have for $h \neq 0$ that

$$
\operatorname{rank}\left(\alpha_{0} \hat{E}\left(t_{\ell}\right)-h \hat{A}\left(t_{\ell}\right)\right)=d_{m}+a_{m}=n-u_{m} .
$$

Thus, if $u_{m} \neq 0$, then the linear system (47) for $x_{\ell}$ is not uniquely solvable. If we set $x_{\ell}=: x_{\ell-1}+\Delta x_{\ell-1}$ we can regularize as follows: We rewrite (47) as

$$
\begin{array}{r}
{\left[\alpha_{0} \hat{E}\left(t_{\ell}\right)-h \hat{A}\left(t_{\ell}\right)\right] \Delta x_{\ell-1}+\left[\alpha_{0} \hat{E}\left(t_{\ell}\right)-h \hat{A}\left(t_{\ell}\right)\right] x_{\ell-1}-} \\
-h \hat{f}\left(t_{\ell}\right)+\hat{E}\left(t_{\ell}\right) \sum_{i=1}^{k} \alpha_{i} x_{\ell-i}=0
\end{array}
$$

and replace this equation by

$$
\left\|\Delta x_{\ell-1}\right\|_{2}=\operatorname{Min} !
$$

subject to the constraint

$$
\begin{aligned}
\|\left[\alpha_{0} \hat{E}\left(t_{\ell}\right)-h \hat{A}\left(t_{\ell}\right)\right] \Delta x_{\ell-1}+\left[\alpha_{0} \hat{E}\left(t_{\ell}\right)-h \hat{A}\left(t_{\ell}\right)\right] x_{\ell-1}- \\
-h \hat{f}\left(t_{\ell}\right)+\hat{E}\left(t_{\ell}\right) \sum_{i=1}^{k} \alpha_{i} x_{\ell-i} \|_{2}=\operatorname{Min} !
\end{aligned}
$$


It is well known (see e.g. [15]) that the solution to (49) is

$$
\begin{aligned}
\Delta x_{\ell-1}=-\left[\alpha_{0} \hat{E}\left(t_{\ell}\right)-h \hat{A}\left(t_{\ell}\right)\right]^{+} & \\
& {\left[\left[\alpha_{0} \hat{E}\left(t_{\ell}\right)-h \hat{A}\left(t_{\ell}\right)\right] x_{\ell-1}-h \hat{f}\left(t_{\ell}\right)+\hat{E}\left(t_{\ell}\right) \sum_{i=1}^{k} \alpha_{i} x_{\ell-i}\right], }
\end{aligned}
$$

where $V^{+}$denotes the Moore-Penrose or generalized inverse of $V$. Note that we use the generalized inverse only for fixing undetermined solution components after having transformed to a suitable normal form. Other approaches using generalized inverses deal with overdetermined problems for the computation of the solution of a uniquely solvable DAE.

As already mentioned above, $\Delta x_{\ell-1}$ is invariant under unitary transformations of $(\hat{E}(t), \hat{A}(t))$ from the left. For a fixed new $x_{\ell}$ we can then determine a residual $R_{\ell}$ by

$$
R_{\ell}=f\left(t_{\ell}\right)+A\left(t_{\ell}\right) x_{\ell}-\frac{1}{h} E\left(t_{\ell}\right) \sum_{i=0}^{k} \alpha_{i} x_{\ell-i}
$$

Observe that here we have used the original quantities $(E(t), A(t), f(t))$. A nonzero residual then indicates that the inhomogeneity is not consistent, i.e. that (18) is violated. Moreover, we obtain consistent initial values $x_{0}$ from the condition

$$
\hat{A}_{2}\left(t_{0}\right) x_{0}+\hat{f}_{2}\left(t_{0}\right)=0
$$

where $\hat{f}_{2}(t)=U_{2}(t)^{*} Z_{2}(t)^{*} g(t)$.

For a given estimate $\tilde{x}_{0}=x_{0}+\delta$, the correction $\delta$ can be determined by the minimization problem $\|\delta\|_{2}=$ Min! subject to the constraint

$$
\left\|\hat{A}_{2}\left(t_{0}\right) \delta+\hat{f}_{2}\left(t_{0}\right)-\hat{A}_{2}\left(t_{0}\right) \tilde{x}_{0}\right\|_{2}=\operatorname{Min} !
$$

i.e. $\delta=\hat{A}_{2}\left(t_{0}\right)^{+}\left(\hat{A}_{2}\left(t_{0}\right) \tilde{x}_{0}-\hat{f}_{2}\left(t_{0}\right)\right)$.

For the simplest BDF-method, the implicit Euler-method, we then have the following algorithm:

Algorithm 1. (Implicit Euler)

Given $\tilde{x}_{0}, t_{0}, h$ as well as $E(t), A(t), f(t)$ together with their derivatives of sufficiently high order.

Step 1: Determine $m, d_{m}, a_{m}, u_{m}$ at $t_{0}$ from the quantities of the corresponding large pairs $\left(M_{\ell}, N_{\ell}\right)$ via Theorem 2.4 and Corollary 2.5.

Step 2: Determine consistent initial values $x_{0}=\tilde{x}_{0}+\delta$ via (54), and check the value of $a_{m}$.

Step 3: Perform one step of the implicit Euler method and evaluate at $t_{1}:=t_{0}+h$.

a) Form $M, N$ at $t=t_{1}$ and determine $m, d_{m}, a_{m}, u_{m}$.

If any of these quantities has changed from the previous steps, then STOP.

b) Determine $\hat{E}, \hat{A}, \hat{f}$ and check $d_{m}, a_{m}$.

c) Compute $\Delta x_{0}$ via (51), check $d_{m}+a_{m}$ and set $x_{1}=x_{0}+\Delta x_{0}$.

d) Compute the residual $R_{1}$ via (52).

e) Write $x_{1}, R_{1}$ and replace $x_{1}$ by $x_{0}$ and $t_{1}$ by $t_{0}$.

f) Repeat a)-e) until satisfied.

For the case $u_{m}=0$ we obtain a smooth differential-algebraic equation with differentiation index

$$
k_{\mathrm{dif}}=\left\{\begin{array}{ll}
0 & \text { if } a_{m}=0 \\
1 & \text { if } a_{m} \neq 0
\end{array} .\right.
$$


In particular one can employ all standard numerical methods for this type of problem, see [19], and the known convergence proofs for these methods are valid.

It remains the question, whether we can generalize these results to the case $u_{m} \neq 0$, where no unique solution exists.

REMARK 1. Following the proof of Theorem 2.6 we can also regularize the problem in the following way: Instead of the pair $(\hat{E}(t), \hat{A}(t))$ in $(42)$ we use

$$
\hat{E}(t)=\left[\begin{array}{c}
\hat{E}_{1}(t) \\
0 \\
0
\end{array}\right], \hat{A}(t)=\left[\begin{array}{c}
\hat{A}_{1}(t) \\
\hat{A}_{2}(t) \\
\hat{A}_{3}(t)
\end{array}\right],
$$

with $\hat{A}_{3}(t)=T_{3}(t)^{*}$. Again there exists a unitary matrix $U_{3}(t)$ with $U_{3}(t) \hat{A}_{3}(t)$ smooth. We then obtain

$$
\hat{r}=d_{m}, \hat{a}=a_{m}+u_{m}, \hat{s}=0, \hat{d}=d_{m}, \hat{u}=0,
$$

i.e. this pair has strangeness index $m=0$ and, since $\hat{u}=0$, there are no more undetermined components. This means in particular that all the convergence results for the standard methods hold, even if the original problem had $u_{m} \neq 0$. We can for example directly apply Theorem 3.2.1 in [2].

REMARK 2. If one wants to fix undetermined components externally, this can be done by assigning the values of $\hat{A}_{3}(t)$ and $\hat{f}_{3}(t)$ and add them to the system in the form

$$
0=\hat{A}_{3}(t) x(t)+\hat{f}_{3}(t)
$$

as an extra $\hat{u}$-block.

4. Numerical Results. We have implemented an experimental code for the approach discussed in the previous section, with both the regularization (49) by means of a least square formulation and the regularization (55) by additional algebraic equations. Note that in the latter case the determination of consistent initial values (54) must include the third row of (55). We refer to these two different regularizations as Methods 1 and 2 in the following. Of course, in the case of $u_{m}=0$ both methods coincide. As integrator we used fixed order, fixed stepsize BDF together with an initial step by step order increase to obtain the initial values for the multistep discretization. A more elaborate code using stepsize and order control and updating procedures to save computation time is currently being developped.

It should be noted that at the heart of this code any discretization method can be introduced. Taking BDF as method of choice we are in the following situation. If we know in advance that the problem has strangeness index 0 and no undetermined solution components, (or equivently differentiation index at most 1), that is if we know that DASSL [28] is applicable, our approach reduces to the discretization used in DASSL, i.e. the computational cost would be identical. If the problem arises from a mechanical multibody system where the differentiation index is known, our approach reduces to the approach implemented in ODASSL [11, 12], i.e. the computational cost would be identical. If, however, no information is available on the strangeness index or the nonuniqueness of solutions, then a considerable computational overhead is necessary to determine this. As indicated in the simplest case of the implicit Euler method, we have to determine the quantities $m, d_{m}, a_{m}, u_{m}$ and filter out the part that we discretize. The computational effort to do this is to compute the local invariants for 
the pairs $\left(M_{\ell}(t), N_{\ell}(t)\right)$ for all sizes $\ell=0, \ldots, m$ using singular value decompositions or QR-decompositions with column pivoting [15] at each discretization point $t_{j}$. As the compuational cost for these decompositions is well known [15], it is easy to determine the costs provided the sizes of the blocks are known. The additional cost will in any case be of the order $O\left(n^{3} m\right)$ per step for strangeness index $m>0$. But if $m$ is not too large this will not change the order of complexity per step, since the solution of the linear system per step will be of this order, too. It is currently under investigation, how to use updating techniques to reduce the computational complexity here. If it turns out that the solution is not unique, then furthermore at each step a least square problem of dimension $n$ has to be solved, which adds more $O\left(n^{3}\right)$ terms but does not change the order of complexity.

In summary, we have that in the cases where codes like DASSL or ODASSL are known to be directly applicable, the computational cost of our approach is exactly the same. Otherwise the cost per step is considerably higher, depending on the sizes of the blocks.

The examples we give below are carried out with our experimental code, for which no big effort was made to optimize computational cost. They are merely given to demonstrate the validity of our theoretical investigations. A practical code, that is currently under development, will allow better comparisons with existing codes, although it should be noted again, that for general high index problems or problems with nonunique solutions, currently there is no alternative to our approach.

In the subsequent examples, we use the following notation:

$$
\begin{aligned}
& E(t), A(t), f(t) \text { - functions specifying the DAE to be solved } \\
& x(t) \quad \text { - general solution of the DAE } \\
& t_{0}, \tilde{x}_{0} \quad \text { - specified initial guess } \\
& h \quad \text { - used stepsize } \\
& N \quad \text { - number of steps to be performed } \\
& k_{\max } \quad-\text { maximal order of BDF to be used } \\
& x_{i} \quad \text { - numerical approximation obtained for } t_{i}=t_{0}+i h
\end{aligned}
$$

EXAmple 1. The DAE given by

$E(t)=\left[\begin{array}{cc}0 & 0 \\ 1 & \eta t\end{array}\right], A(t)=\left[\begin{array}{cc}-1 & -\eta t \\ 0 & -1-\eta\end{array}\right], f(t)=\left[\begin{array}{c}e^{-t} \\ 0\end{array}\right], x(t)=\left[\begin{array}{c}(1-\eta t) e^{-t} \\ e^{-t}\end{array}\right]$

is used in the literature to show the possible instability of the implicit Euler method when directly applied to (1). In the above case, this occurs for $\eta<-0.5$, see e.g. [2]. Taking $\eta=-1$ and

$$
t_{0}=0, \tilde{x}_{0}=(0,0)^{T}, h=0.01, N=100, k_{\max }=1,
$$

we obtained

$$
m=1, d_{m}=0, a_{m}=2, u_{m}=0
$$

and

$$
x_{0}=(1.0000,1.0000)^{T}, x_{N}=(0.7358,0.3679)^{T} .
$$

This shows that the instabilities are due to a wrong discretization in the sense that direct discretization of (1) in the case $m \neq 0$ ignores the invariants of the given problem. 
EXAMPLE 2. An undetermined solution component occurs in the DAE given by

$$
E(t)=\left[\begin{array}{cc}
-t & t^{2} \\
-1 & t
\end{array}\right], A(t)=\left[\begin{array}{cc}
-1 & 0 \\
0 & -1
\end{array}\right], f(t)=\left[\begin{array}{l}
0 \\
0
\end{array}\right], x(t)=\left[\begin{array}{c}
t \varphi(t) \\
\varphi(t)
\end{array}\right],
$$

see e.g. $[16,20]$. Using

$$
t_{0}=0, \tilde{x}_{0}=(1,1)^{T}, h=0.01, N=100, k_{\max }=6,
$$

we got

$$
m=1, d_{m}=0, a_{m}=1, u_{m}=1
$$

and

$$
x_{0}=(0.0000,1.0000)^{T}, x_{N}=(0.7048,0.7048)^{T}
$$

for Method 1 whereas for Method 2 the trivial solution was realized. For $h \rightarrow 0$ the numerical solution of Method 1 at $t=1$ seems to tend to $\left(\frac{1}{2} \sqrt{2}, \frac{1}{2} \sqrt{2}\right)^{T}$.

EXAMPle 3. In [31] we find a multibody problem of differentiation index 2 with $n=7$. A consistent initial condition is given by $\left(t_{0}, x_{0}\right)=(0,0)$. Both for strong and weak coupling the presented results could be reproduced. The obtained invariants were

$$
m=1, d_{m}=5, a_{m}=2, u_{m}=0 .
$$

EXAmple 4. A complete description of a nonlinear as well as a linearized truck model including consistent initial values can be found in [30]. The problem of size $n=23$ is known to be of differentiation index 3. Again the reported solution could be reproduced with invariants

$$
m=2, d_{m}=20, a_{m}=3, u_{m}=0 .
$$

Solving this problem, two difficulties arose. First, the obtained rank decisions were found to be not reliable due to a bad scaling of the equations. Rescaling $E(t)$ which is diagonal in this example to identity in the nonzero part then gave satisfactory results. Second, instabilities of the multistep scheme occured due to large eigenvalues near the imaginary axis. In this case, reducing the maximal order of the BDF to $k_{\max }=3$ led to a stable discretization.

Examples 3 and 4 are so-called multibody problems arising as Euler-Lagrange equations for motion under constraints. These equations exhibit a very special structure. In particular, the index is known in advance. It is clear that for an efficient numerical treatment this structure must be utilized. See e.g. [12, 24] for sophisticated methods in this area of research. Note also that general purpose solvers like given in $[10,17,28]$ cannot be applied to Examples 1 and 2 since they are restricted to $k=1$ which here implies $m=0$.

5. Conclusion. We have presented a new class of discretization methods for linear differential-algebraic equations with variable coefficients. The key idea is the extraction of a strangeness free system from the original equation and its derivatives, plus a regularization of the part of the system corresponding to undetermined components. The discretization is then performed by applying BDF-methods to this extracted system. We have presented some numerical examples, which show that the new methods are able to handle systems of arbitrary index even having undetermined components. 


\section{REFERENCES}

[1] A. Barrlund, Constrained least squares methods for linear timevarying DAE systems, Numerische Mathematik, 60 (1991), pp. 145-161.

[2] K. Brenan, S. Campbell, and L. Petzold, Numerical Solution of Initial-Value Problems in Differential Algebraic Equations, Elsevier, North Holland, New York, NY, 1989.

[3] S. L. CAmpBell, A general form for solvable linear time varying singular systems of differential equations, SIAM Journal Mathematical Analysis, 18 (1987), pp. 1101-1114.

[4] - Local realizations of time varying descriptor systems, in Proceedings of the 26th IEEE Conference on Decision and Control, 1987, pp. 1129-1130.

[5] - The numerical solution of higher index linear time varying singular systems of differential equations, SIAM J. Sci. Stat. Comput., 6 (1988), pp. 334-348.

[6] - Uniqueness of completions for linear time varying differential algebraic equations, Linear Algebra and its Applications, 161 (1992), pp. 55-67.

[7] S. L. Campbell and E. Gripentrog, Solvability of general differential algebraic equations, tech. report, Dept. of Mathematics, North Carolina State University, Raleigh, North Carolina USA, 1994. to appear in SIAM J. Sci. Stat. Comput.

[8] S. L. Campbell and E. Moore, Progress on a general numerical method for nonlinear higher index DAEs, Tech. Report NA.0292021, Dept. of Mathematics, North Carolina State University, Raleigh, North Carolina USA, 1992.

[9] - Constraint preserving integrators for general nonlinear higher index DAEs, tech. report, Dept. of Mathematics, North Carolina State University, Raleigh, North Carolina USA, 1994. to appear in Numerische Mathematik.

[10] P. Deuflhard, E. Hairer, And J. ZugCK, One-step and extrapolation methods for differentialalgebraic systems, Numerische Mathematik, 51 (1987), pp. 501-516.

[11] C. FüHRER, Differential-Algebraische Gleichungssysteme in mechanischen Mehrkörpersystemen, Dissertation, Technische Universität München, München, FRG, 1988.

[12] C. Führer AND B. J. Leimkuhler, Numerical solution of differential-algebraic equations for constrained mechanical motion, Numerische Mathematik, 59 (1991), pp. 55-69.

[13] C. W. GEAR, Differential-algebraic index transformations, SIAM J. Sci. Stat. Comput., 9 (1988), pp. 39-47.

[14] C. W. Gear and L. R. Petzold, Differential-algebraic systems and matrix pencils, in Matrix Pencils, B. Kågstrom and A. Ruhe, eds., Springer Verlag, Berlin, 1983, pp. 75-90.

[15] G. H. Golub and C. F. Van Loan, Matrix Computations, The Johns Hopkins University Press, Baltimore, Maryland, 2nd ed., 1989.

[16] E. Griepentrog And R. März, Differential-Algebraic Equations and Their Numerical Treatment, Teubner Verlag, Leipzig, 1986.

[17] E. Hairer, C. Lubich, and M. Roche, The Numerical Solution of Differential-Algebraic Systems by Runge-Kutta Methods, Springer-Verlag, Berlin, 1989.

[18] E. Hairer, S. Nørsett, And G. Wanner, Solving Ordinary Differential Equations I, SpringerVerlag, Berlin, 1987.

[19] E. Hairer And G. Wanner, Solving Ordinary Differential Equations II, Springer-Verlag, Berlin, 1991.

[20] B. Hansen, Comparing different concepts to treat differential-algebraic equations, Tech. Report 220, Sektion Mathematik, Humboldt Universität Berlin, Berlin, FRG, 1989.

[21] P. Kunkel and V. Mehrmann, Numerical solution of differential-algebraic Riccati equations, Linear Algebra and its Applications, 137/138 (1990), pp. 39-66.

[22] — Smooth factorizations of matrix valued functions and their derivatives, Numerische Mathematik, 60 (1991), pp. 115-132.

[23] - Canonical forms for linear differential-algebraic equations with variable coefficients, Tech. Report 69, Institut für Geometrie und Praktische Mathematik, RWTH Aachen, Templergraben 55, D-5100 Aachen, FRG, to appear in J. Comp. Appl. Math., 1992.

[24] C. Lubich, U. Nowak, U. Pöle, And C. Engstler, MEXX-Numerical software for the integration of constrained mechanical multibody systems, Tech. Report SC 92-12, ZIB, Berlin, 1992.

[25] R. MÄrz, Numerical methods for differential-algebraic equations I: Characterizing DAEs, Tech. Report 91-32/I, Fachbereich Mathematik, Humboldt Universität Berlin, Berlin, FRG, 1991.

[26] — Numerical methods for differential-algebraic equations II: Numerical integration methods, Tech. Report 91-32/II, Fachbereich Mathematik, Humboldt Universität Berlin, Berlin, FRG, 1991

[27] V. Mehrmann, The Autonomous Linear Quadratic Control Problem, Springer-Verlag, Berlin, 1991. 
[28] L. R. Petzold, A description of DASSL: A differential/algebraic systems solver, in Scientific Computing, R. S. S. et al., ed., North-Holland, Amsterdam, 1983, pp. 65-68.

[29] P. Rabier and W. Rheinboldt, Classical and generalized solutions of time-dependent linear differential algebraic equations, tech. report, Dept. of Mathematics and Statistics, Univ. of Pittsburgh, Pittsburgh, PA 15260, USA, 1993.

[30] B. Simeon, F. Grupp, C. Führer, And P. Rentrop, A nonlinear truck model and its treatment as a multibody system, Tech. Report TUM-M9204, Mathematisches Institut, TU München, München, FRG, 1992.

[31] T. Schmidt and M. Hou, Rollringgetriebe. Internal Report, Sicherheitstechnische Regelungsund Meßtechnik, Bergische Universität, GH Wuppertal, Wuppertal, FRG, 1992. 